\title{
El Torno de Pedal
}

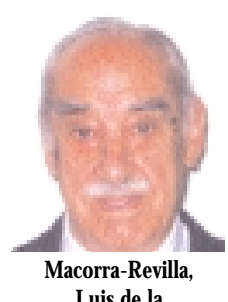

The foot pedal dental engine

Macorra-Revilla, Luis de la* Conzález- Ruiz, Ignacio **

*Médico Estomatólogo. Director del Museo de Odontología de la U.C.M. **Odontólogo.

\section{Resumen}

El Torno de Pedal ha sido y en muchos lugares del mundo continúa siendo una herramienta básica para el desarrollo de la odontología.

Se describen desde un punto de vista mecánico los elementos que generalmente constituyen un Torno de Pedal.

Finalmente se describe su funcionamiento.

Palabras clave: Torno de Pedal, Morrison, J.B, Sistemas rotatorios de fresas.

\section{Abstract}

The foot pedal dental engine was -and still is in a lot of places around the wolda basic tool for the development of dentistry. The parts that generally make up a foot pedal dental engina are described from a mechanical point of view, as well as its performance.

Key words: Foot pedal dental engine, Morrison, J.B. rotary drill systems.

\begin{tabular}{ccc}
\hline Fecha recepción & Fecha última revisión & Fecha aceptación \\
$13-12-2002$ & $5-02-2003$ & $14-03-2003$ \\
\hline
\end{tabular}

BIBUD [1138-123X (2003)8:3; mayo-junio 245-348]

Macorra-Revilla L dela, González-Ruiz I. El Torno de Pedal. RCOE 2003;8(3):305-314. 


\section{Introducción}

Muchos son los museos y consultas del mundo donde duerme su olvido un torno de pedal, lo que no es sino una injusticia para esta magnífica herramienta, que durante casi un siglo prestó un inmejorable servicio.

Fue precisamente un odontólogo americano, J.B. Morrison (fig.1), que educado en el conocimiento de la mecánica, patentó el 7 de febrero de $1871^{1}$ un sistema rotatorio que permitía un trabajo más rápido y fácil que el que hasta entonces se lograba, bien por las fresas giradas por medio de la cuerda de un arco, bien por el sistema de Arquímedes, que conseguía la rotación de la fresa, deslizando un cursor con mango en el tornillo salomónico de una «bailarina» (fig. 2).

Consistía «el invento» en un volante que se hacía girar por medio de un pedal y una biela, giro que era transmitido por un sistema de correa y poleas a la fresa, martillo de orificar, léntulos, ensanchadores, trócares, etc.

Resulta digno de admiración, el hecho de que durante todo el reinado del Torno de Pedal, la idea básica de Morrison se mantuvo con pocas variaciones aunque los distintos fabricantes fueron incorporando con el paso de los años pequeñas mejoras que seguidamente intentaremos reflejar.
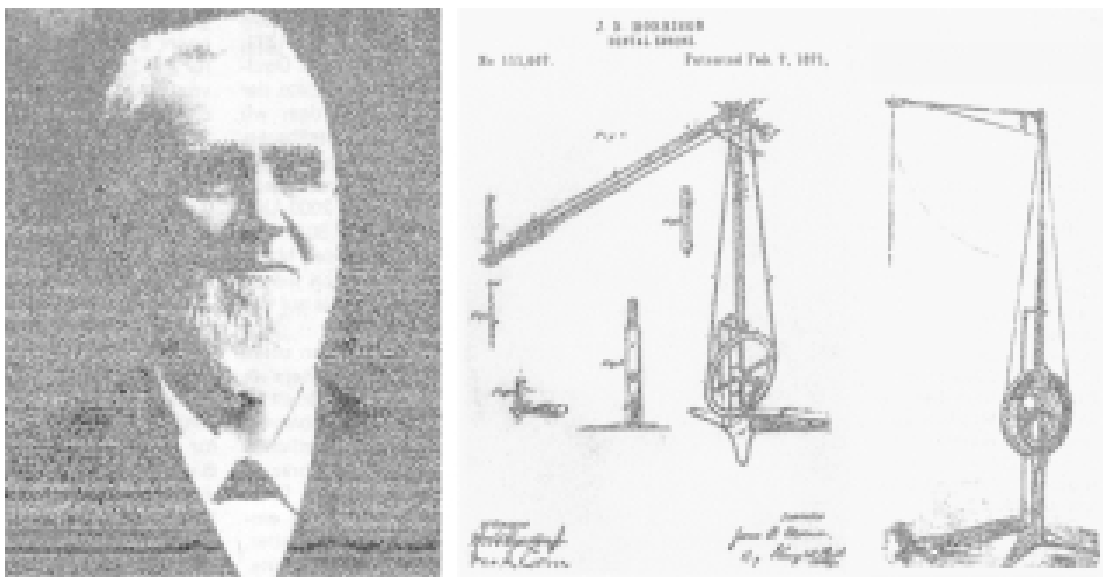

Figura 1: J.B. Morrison, inventor del Torno de Pedal.

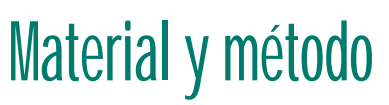

Se han empleado para este estudio un total de cincuenta y siete Tornos de Pedal que proceden de dos fuentes distintas:

1) Treinta y tres tornos de pedal pertenecientes a museos, colecciones particulares y a colegas:

- Siete del Museo de la Facultad de Odontología UCM (Madrid).

- Seis del Museo Pedro Borja (Gandía). - Ocho de la Colección Privada del Dr. de la Macorra (Madrid).

- Seis de propiedad particular, pertenecientes a:

- Dra. Elena Barbería (Madrid).

- Dr. José Carlos de la Macorra. (Madrid).

- Dr. Javier Tapias (Madrid).
- Dra. Fabiola Kessler (Madrid).

- A. Martín (Madrid).

- Dr. Hugo Rivera (Madrid).

2) Veinticuatro imágenes procedentes de catálogos y libros de la época (que se citan en el índice bibliográ(fico) $)^{2-7}$.

La simple descripción de los elementos componentes del Torno de Pedal nos ha parecido la manera más sencilla y amena de presentar a los lectores esta elemental máquina. Lectores que podemos clasificar en cuatro grupos:

1ㅇ) Aquellos jóvenes que ignoran su existencia.

2ํ) Profesionales cuyo único conocimiento sobre el torno, sea su mera existencia.

3) Profesionales que no solamen-
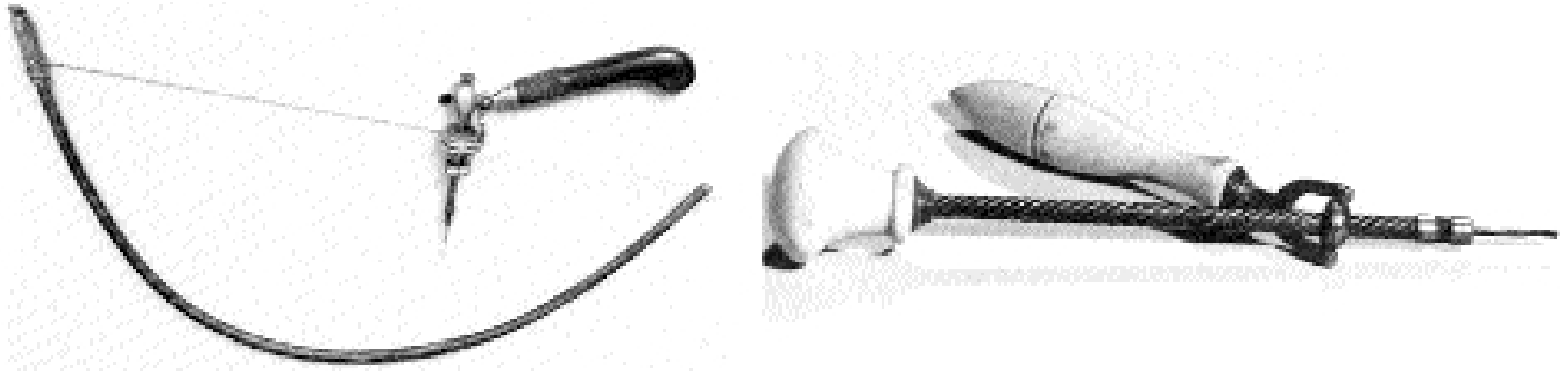

Figura 2: Primitivos Tornos: de Arco de «Bailarina».

RCOE, 2003, Vol 8, №3, 305-314 


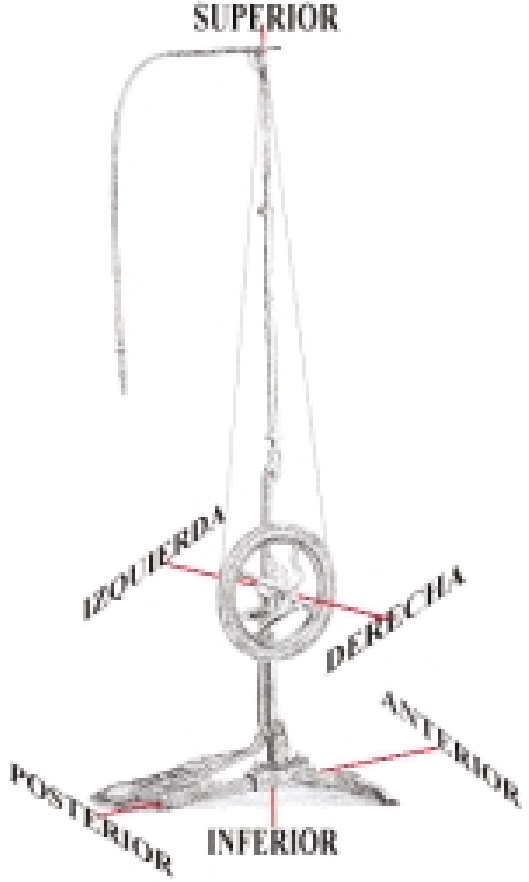

Figura 3: Orientación en el espacio del Torno de Pedal.

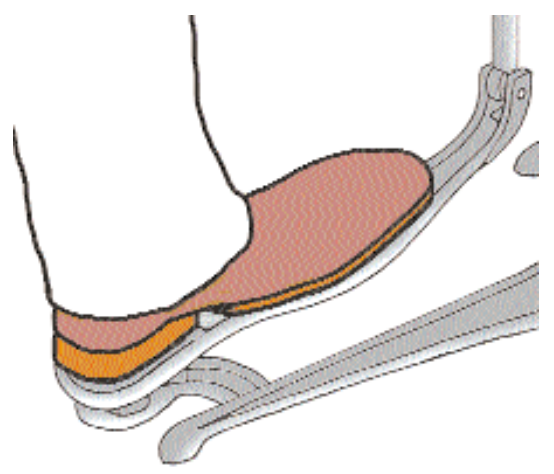

Figura 4: El pie del profesional es el que sirve para la orientación.

te conocen su existencia sino que poseen un torno de pedal como mero objeto decorativo en sus consultas.

4) Profesionales que hayan tenido el privilegio de usar esta encantadora herramienta, ya que en España se puede decir que desapareció su uso después de los primeros años del cua-

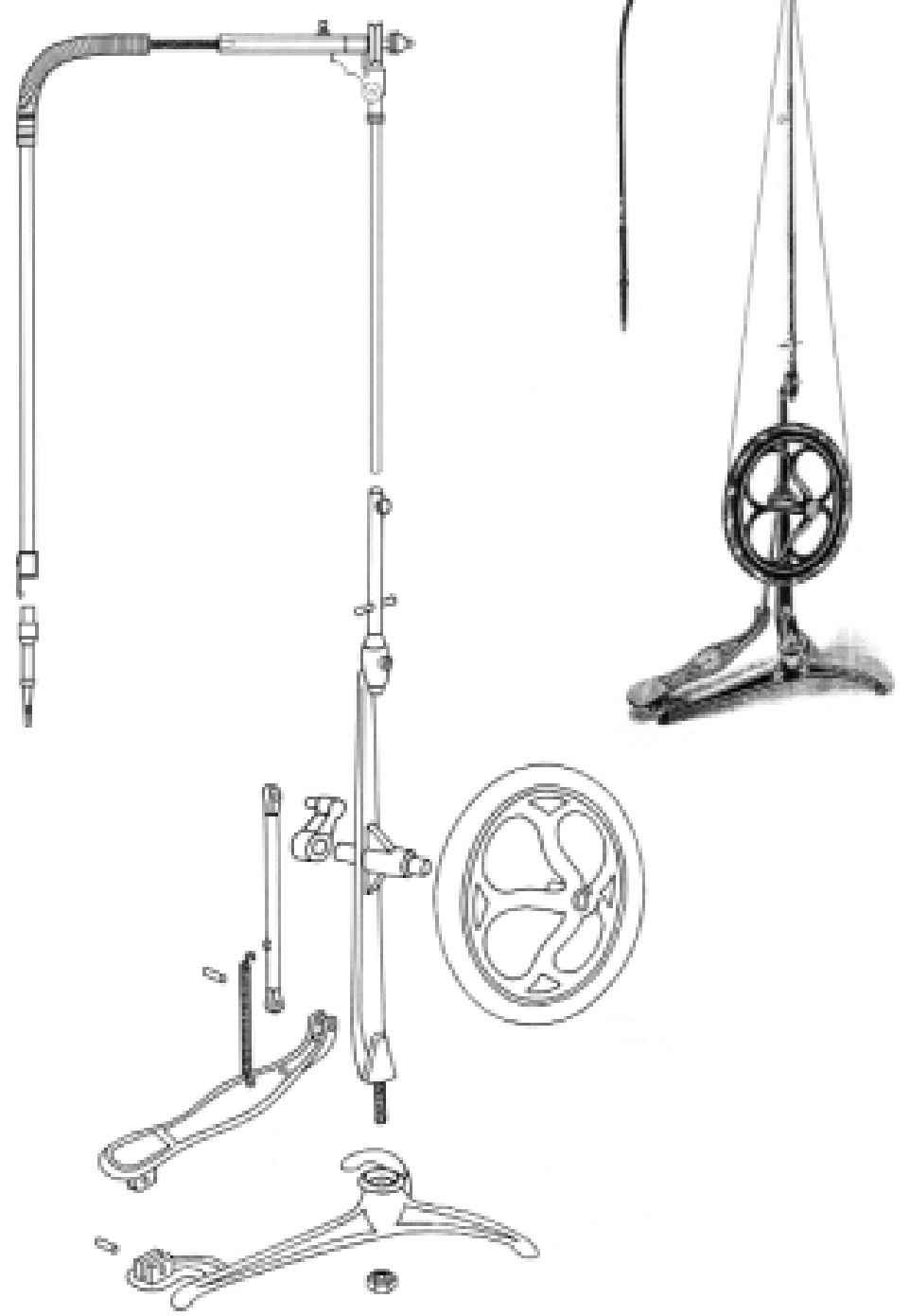

Figura 5: Partes que componen el Torno de Pedal.

renta, al cesar las restricciones eléctricas de aquella época.

Siendo preciso indicar que varios años antes de la Guerra Civil (19361939) el torno eléctrico ya había desbancado al torno de pedal y que fue la propia guerra la que circunstancialmente devolvió parte de su protagonismo a nuestra querido motor dental (Dental Engine).

\section{Partes}

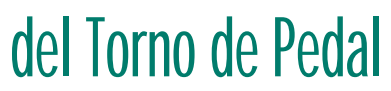

Con objeto de que la descripción de las partes componentes de un Torno de Pedal sea fácilmente comprensible, debemos, en primer lugar, darle una orientación en el espacio (fig. 3). De este modo, consideraremos parte 


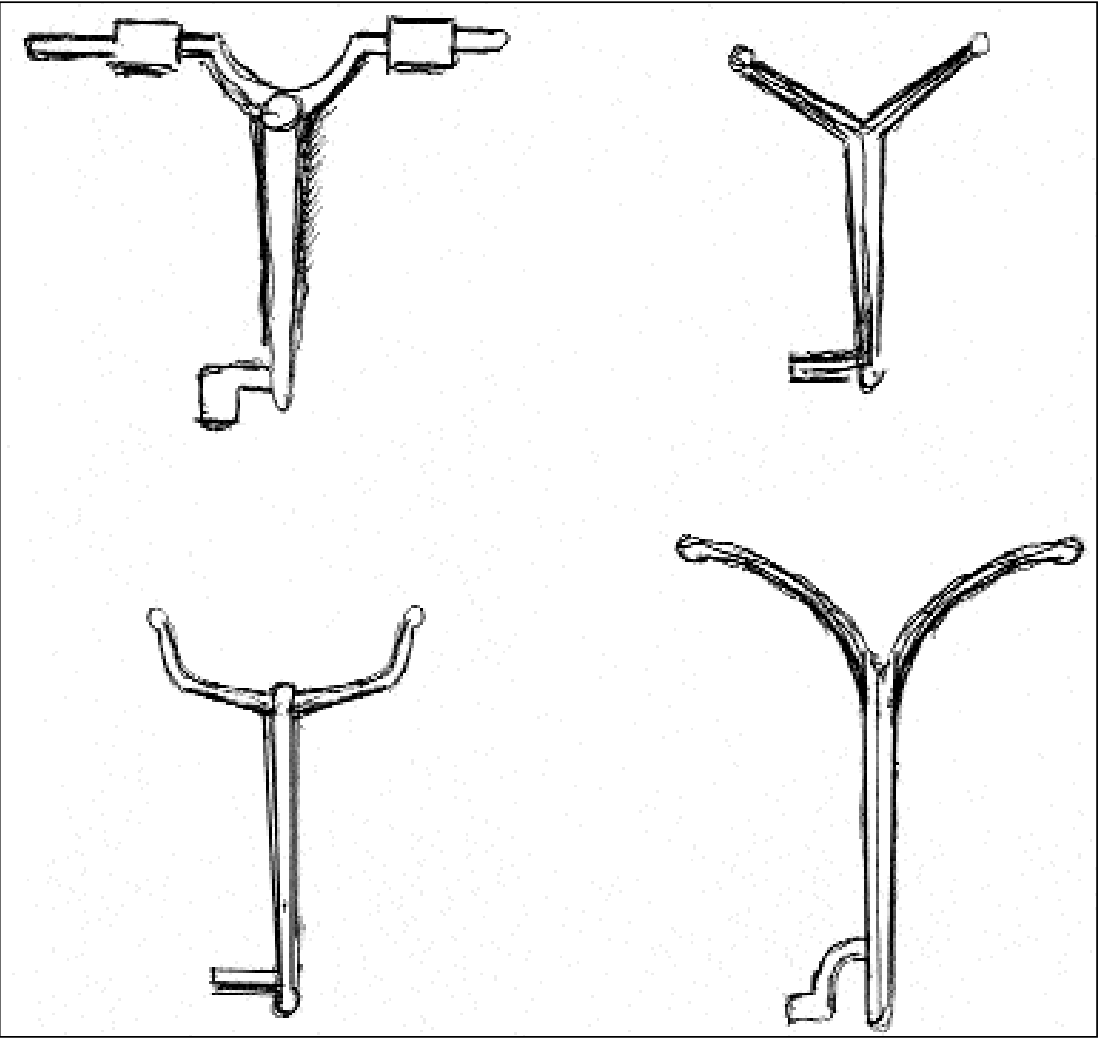

Figura 6: Peanas o trípodes.

inferior la correspondiente al trípode o peana que se apoya en el suelo y parte superior, la opuesta.

En lo referente a posterior, anterior, derecha e izquierda, será el pie del profesional la brújula que nos oriente (fig. 4).

Una vez orientados, pasamos a describir las distintas partes de que se compone (fig. 5) un torno de pedal que consideramos prototipo identificable con la gran mayoría de tornos existentes.

\section{Peana o trípode}

Generalmente de hierro fundido, adopta la solución de contar con tres puntos de apoyo en el suelo (trípode), lo que le permite una mejor adaptación sobre el mismo.
En su brazo posterior dispone de un acoplamiento para el eje del pedal. La disposición y forma de los otros dos brazos varía según el fabricante y mostramos a continuación las variantes más comunes (fig. 6).

En su parte central se encuentra el sistema de fijación del soporte, existiendo en este punto diversas soluciones entre las que figuran: vástago pasante con rosca y tuerca de fijación, vástago ajustado y tornillo de presión, dos tornillos de sujeción, etc.

\section{Soporte}

Pieza central del sistema, generalmente de hierro fundido que, sujetándose en la confluencia de los tres brazos del trípode o peana, se dirige verticalmente hacia arriba a modo

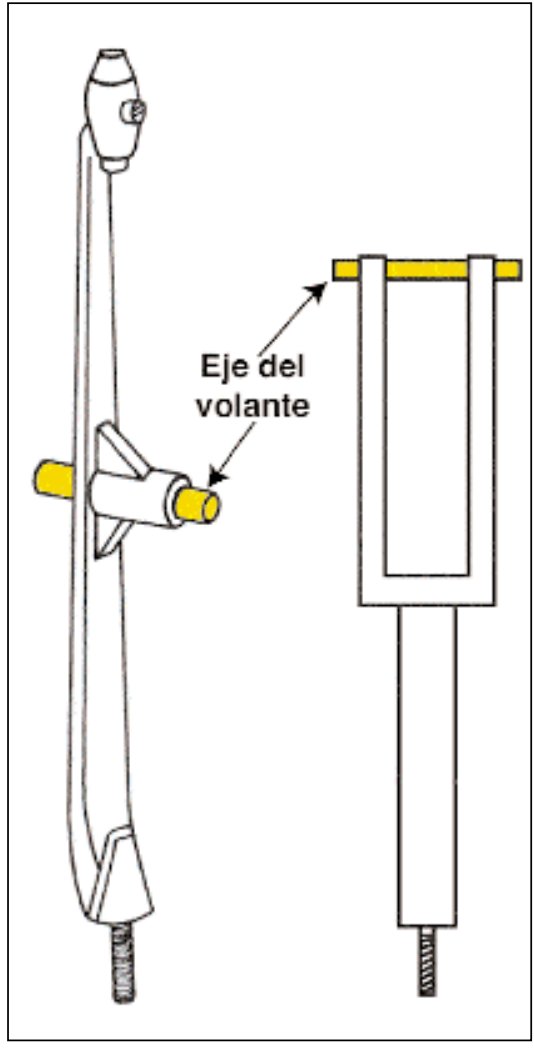

Figura 7: Soportes: sencillo y horquilla.

de columna vertebral, alojando en su parte media un eje horizontal en sentido transversal (eje del volante) y en su parte superior la varilla o caña.

Existen dos tipos de soportes en cuanto a su forma (fig. 7):

1) El soporte de horquilla (cuyos brazos llegan al eje horizontal del volante, para darle doble apoyo, eliminando el «cantilever»).

2) El soporte simple o monobrazo.

El soporte monobrazo presenta dos quiebros orientados hacia el volante (uno inferior y otro superior) que permiten alojar el volante, quedando el plano de trabajo de éste sobre el centro de la base de estabilidad del aparato, lo que favorece que el momento giroscópico del volante 


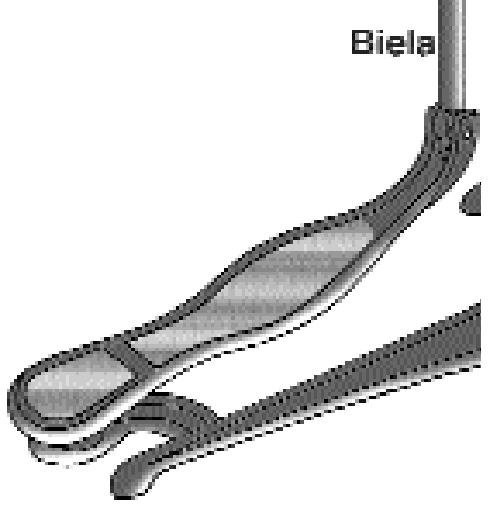

Figura 8: Pedal.

coincida con el plano central axial del sistema, dándole mayor estabilidad.

El tramo del soporte que denominaremos interquiebro por estar situado entre los citados quiebros, puede adoptar las siguientes formas:

a) Ser recto, unido en su parte media con el alojamiento del eje en cantilever o voladizo, al que se refuerza con sendas escuadras fundidas superior e inferior.

b) Ser recto con quiebro central en puente, que permite unirse en su parte media a la parte central del alojamiento del eje volante, suprimiendo el cantilever y sus escuadras.

Tanto el soporte de horquilla como

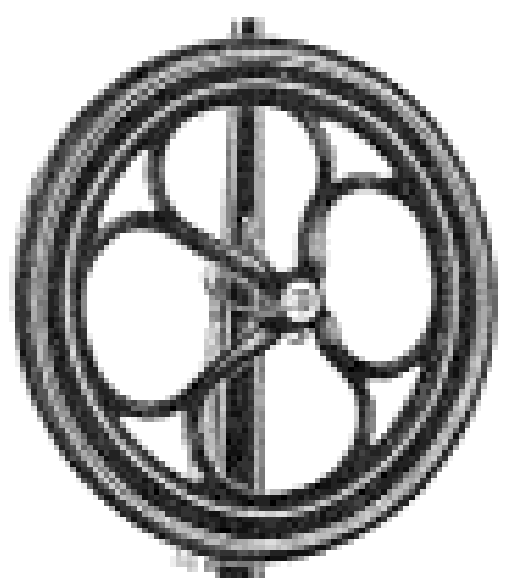

el soporte monobrazo pueden ser rígidos o articulados.

Los soportes articulados tienen el propósito de poder acercar la pieza de mano a la boca del paciente por medio de la inclinación de la varilla, existiendo diversos mecanismos para conseguir dicha inclinación y su fijación en una posición concreta.

En la parte superior del soporte, en algunos modelos, es posible encontrar dos apéndices horizontales que sirve a modo de agarradera para el transporte del instrumento.

\section{Pedal}

Pieza de hierro fundido de forma apropiada para alojar el pie del operador que activa el sistema, que articulada en su parte postero-inferior por medio de un eje en el brazo posterior del trípode, se dirige hacia delante y hacia arriba, articulándose con la parte inferior de la biela o pie de biela (fig. 8).

Generalmente se encuentra situado en la parte izquierda del trípode o peana, pero en algunos modelos, presumiblemente para zurdos, se encuentra a la derecha de la misma.

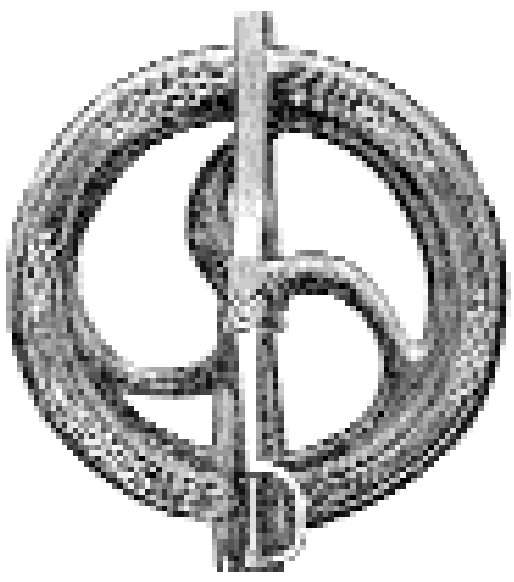

Figura 9: Volantes.
Cuando el sistema se encuentra inactivo, el pedal presenta una inclinación de abajo a arriba y de tacón a puntera, correspondiente a la posición de arranque.

La superficie del pedal donde el profesional apoya su pie, suele llevar algún sistema para evitar el deslizamiento, como son por ejemplo gomas o dibujos realizados sobre la pieza de fundición.

\section{Volante}

Pieza de forma circular, generalmente fabricada en hierro fundido, con la mayor parte de su peso ubicado en su periferia, con el propósito de aumentar su momento de inercia de rotación y con un canal periférico central o garganta, para alojar la correa de transmisión o correa sin fin.

Existen diferentes modelos en cuanto a la forma de sus radios, predominando los círculos (tres o cuatro) inscritos en el espacio interior de la masa periférica del volante (fig. 9).

Los radios pueden también ser rectos del centro a la periferia o curvados, variando ligeramente en su número, siendo lo más frecuente tres o cuatro.

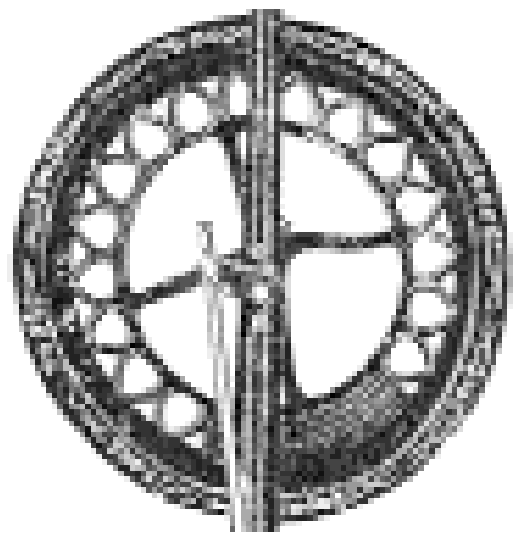

RCOE, 2003, Vol 8, №3, 305-314 


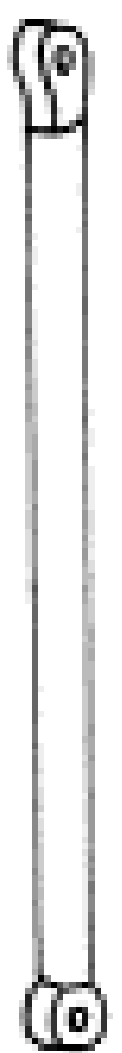

Figura 10: Bielas: rígida $y$ flexible.

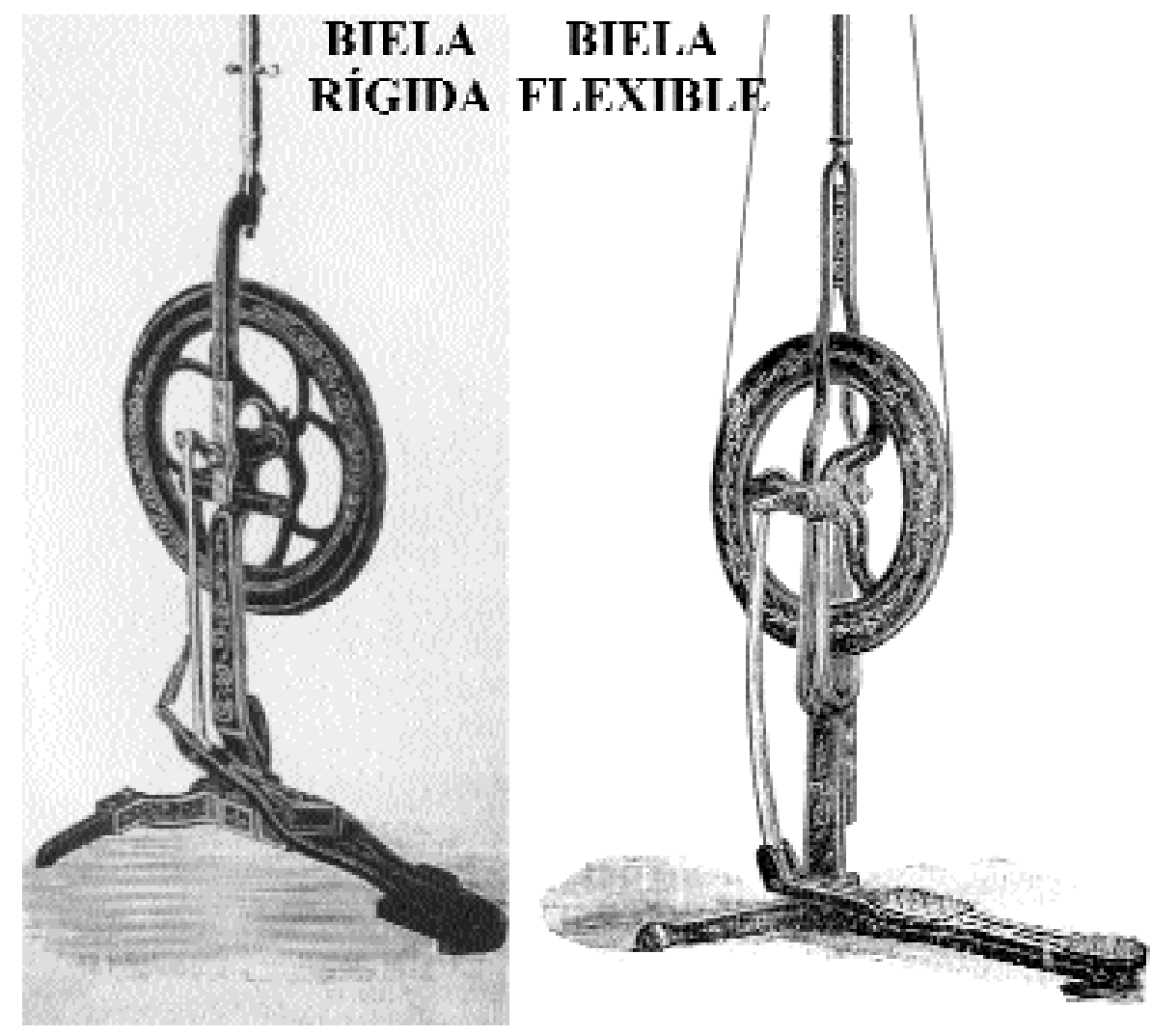

Figura 11: Bielas flexible y rígida montadas en el Torno de Pedal.
El volante puede ser a su vez plano o aparaguado, pero siempre solidario con el eje horizontal de rotación que se encuentra en la parte media del soporte (eje del volante).

El volante plano es el que se emplea con el soporte de horquilla y el aparaguado con el soporte simple o monobrazo, aunque es posible encontrar volantes planos en soportes monobrazo.

\section{Biela}

Pieza metálica destinada a transmitir el movimiento rectilíneo de ascenso y descenso de la parte delantera del pedal a la manivela (fig. 10).

El extremo superior de la biela se denomina cabeza de biela y el extremo inferior pie de biela.

La cabeza de la biela suele alojar un casquillo de bronce que favorece el giro sobre la muñequilla de la manivela.

La biela también puede ir de la punta del pedal a un vástago o bulón, que saliendo perpendicular al plano del volante, se encuentra en uno de sus radios y a determinada distancia del eje de giro.

Existen dos modalidades de biela:

- Rígida $\left\{\begin{array}{l}\text { Recta. } \\ \text { Curva. }\end{array}\right.$

- Flexible.

Las bielas de tipo rígido, exigen algún sistema que evite el punto muerto de giro. Esto se consigue o porque el volante presenta un peso adicional excéntrico, o por la existencia de un muelle que con su elasticidad, se encarga de salvar la posición de punto muerto.

Tanto las bielas rectas como las curvas, presentan dos modalidades de su apoyo al pedal:

1) La unión pedal-biela se efectúa entre el pie de la biela y el extremo anterior del pedal.

2) La unión pedal-biela está en la biela a unos centímetros alejada de su pié, quedando el pie de la biela por debajo del plano del pedal.

Las bielas de tipo flexible están constituidas por un fleje u hoja de ballesta, que uniéndose de forma rígi- 

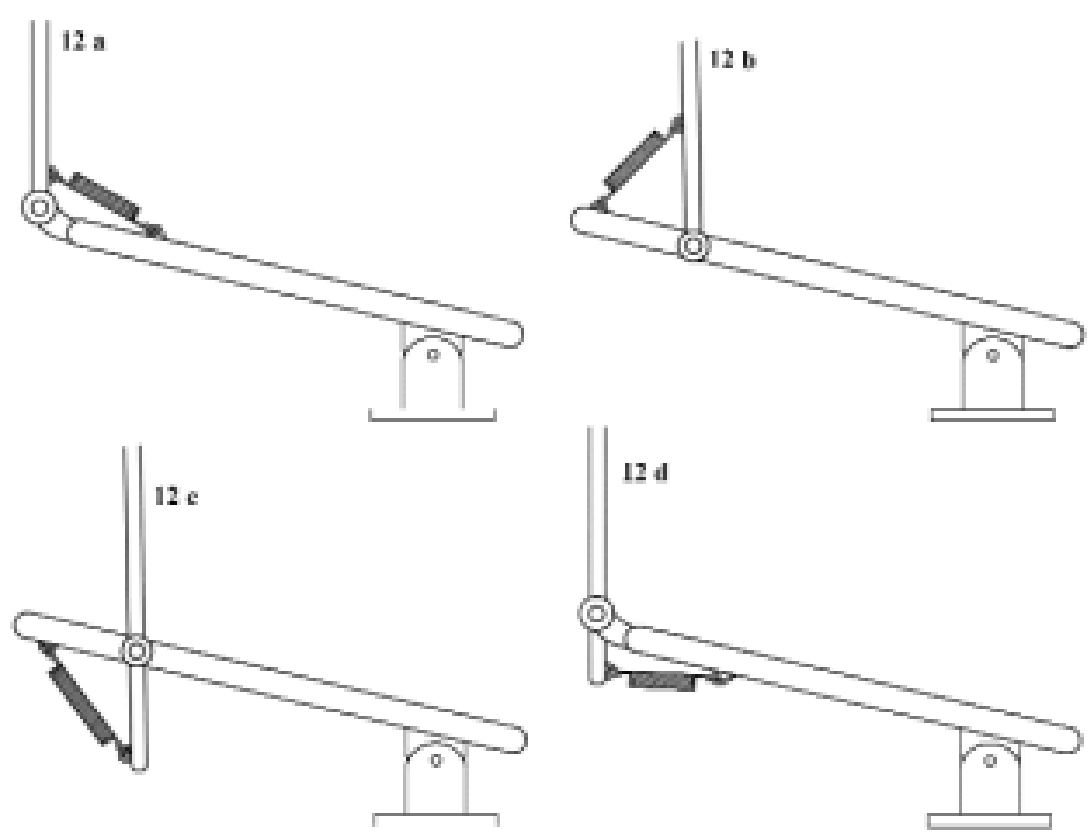

$12 \mathrm{~d}$

Figura 12: Muelles: a) por encima del pedal y detrás de la biela; b) Por encima del pedal y delante de la biela; c) Por debajo del pedal y delante de la biela; d) por debajo del pedal y detrás de la biela.

da con la punta del pedal, ascienden hasta la muñequilla de la manivela, que con su giro obliga a la biela a flexionarse y ésta, con su elasticidad, tiende a eliminar el punto muerto (fig. 11).

\section{Muelle}

Elemento elástico de acero de forma espiral, situado entre la biela y el pedal, que pudiendo extenderse y deformarse con el estímulo de tensión, es capaz de recuperar su postura inicial y así salvar el punto muerto, favoreciendo la posición de arranque.

Este dispositivo del muelle sólo se emplea para las bielas rígidas, ya que en las bielas de tipo flexible es su propia característica de flexibilidad la que realiza la función de recuperación que ejerce el muelle. Esta misma función se consigue con el contrapeso excéntrico en el volante, como ya se ha comentado. Resumiendo, existen tres maneras de favorecer la posición de arranque del pedal: muelle, biela flexible y contrapeso.

Cuatro son las posiciones que puede ocupar el muelle con respecto al conjunto pedal-biela:

a) Por encima del pedal y detrás de la biela (fig.12 a).

b) Por encima del pedal y delante de la biela (fig.12 b).

c) Por debajo del pedal y delante de la biela (fig. $12 \mathrm{c}$ ).

d) Por debajo del pedal y detrás de la biela (fig. $12 \mathrm{~d}$ ).

\section{Manivela}

Pieza de acero solidaria con el eje horizontal (eje del volante), que presenta un bulón o muñequilla, excéntrico y paralelo a dicho eje, que se articula con la cabeza de biela de tal

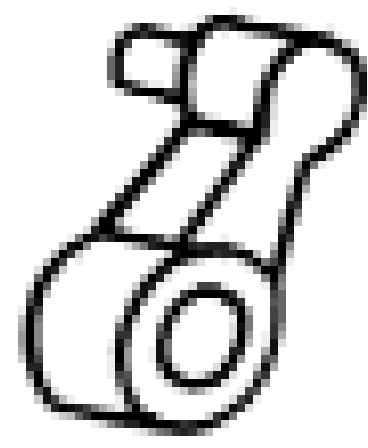

Figura 13: Manivela

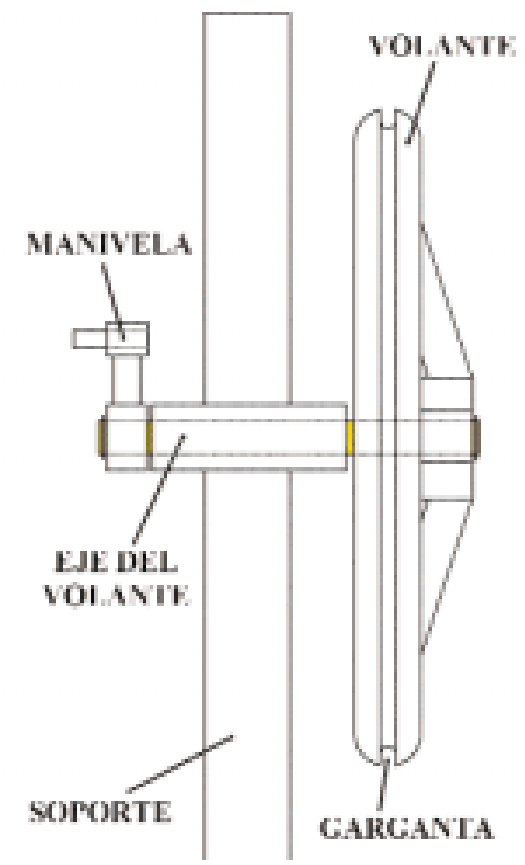

Figura 14: Eje horizontal

forma y manera que, permitiendo y recibiendo el movimiento circular de la cabeza de la biela produce en el eje horizontal del volante su movimiento de rotación (fig. 13).

\section{Eje horizontal (Eje del volante)}

Cilindro de acero de uno a dos centímetros de diámetro, alojado en la parte media del soporte en el plano horizontal y en sentido transversal y 


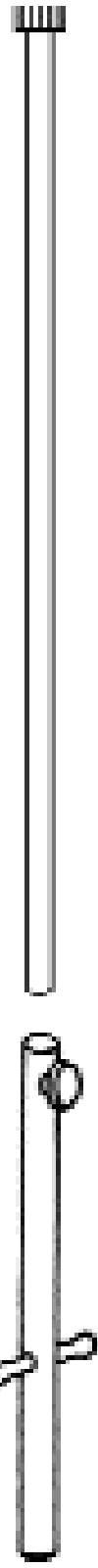

Figura 15: Varilla o caña. 15 a) Apéndices horizontales para el transporte del torno.

solidario en uno de sus extremos con el volante y en el otro con la manivela.

Es sorprendente el hecho de que en algunos casos, no presente rodamientos ni casquillos de bronce (fig. 14).

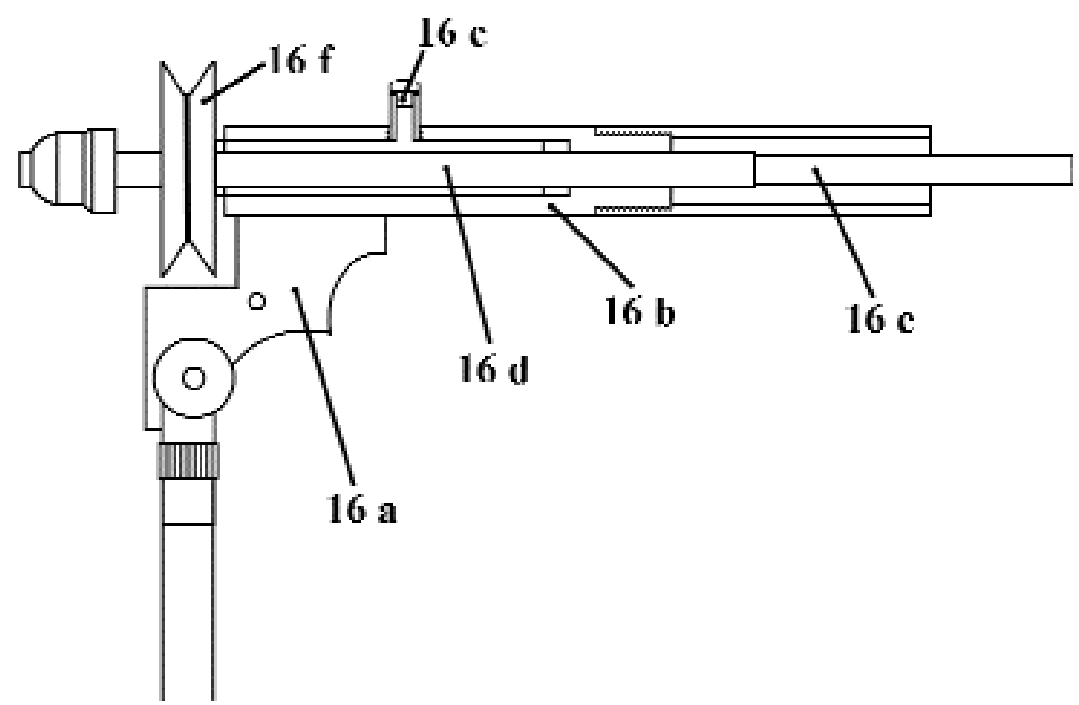

Figura 16: Bisagra: 16 a) Elemento articular. 16 b) Brazo horizontal. 16 c) Engrasador. 16 d) Eje acanalado. 16 e) Cable. 16 f) Rueda metálica giratoria con garganta en su periferia.

\section{Varilla o caña}

Tubo cilíndrico metálico recto, que sujeto en la parte superior del soporte por medio de un tornillo de presión, se dirige verticalmente hacia arriba, donde aloja la base de la bisagra (fig. 15).

Presenta un dispositivo que permite aumentar su longitud para obtener una adecuada tensión de la correa de transmisión. Pudiendo encontrarse dicho dispositivo en su parte inferior o en su parte media.

Puede presentar a la altura de su parte inferior dos apéndices horizontales (fig. 15 a), destinados a servir a modo de agarradera para el transporte del Torno de Pedal. Aunque, como ya se ha indicado, en algunos modelos estos apéndices se encuentran en la parte superior del soporte, estando destinados al mismo fin.

\section{Bisagra}

Elemento articular situado en el extremo superior de la varilla, que permite al brazo horizontal plegarse sobre la misma y así facilitar el transporte (fig. $16 \mathrm{a}$ ).

La bisagra está compuesta a su vez por dos brazos, uno inferior que se une con la varilla y otro superior que sirve de soporte al brazo horizontal.

Su brazo inferior que sirve de nexo de unión con la varilla dispone de un elemental sistema de muelle para fijar la articulación en diferentes posiciones.

\section{Brazo horizontal}

Tubo cilíndrico metálico unido al brazo superior de la bisagra, que aloja en su interior un eje acanalado, permitiendo el giro del mismo (fig. 16 b).

El brazo horizontal constituye una prolongación o alargadera del brazo superior de la bisagra.

Puede presentar un engrasador (fig. $16 \mathrm{c}$ ).

\section{Eje acanalado}

Elemento giratorio y acanalado, como su propio nombre indica (fig. 16 


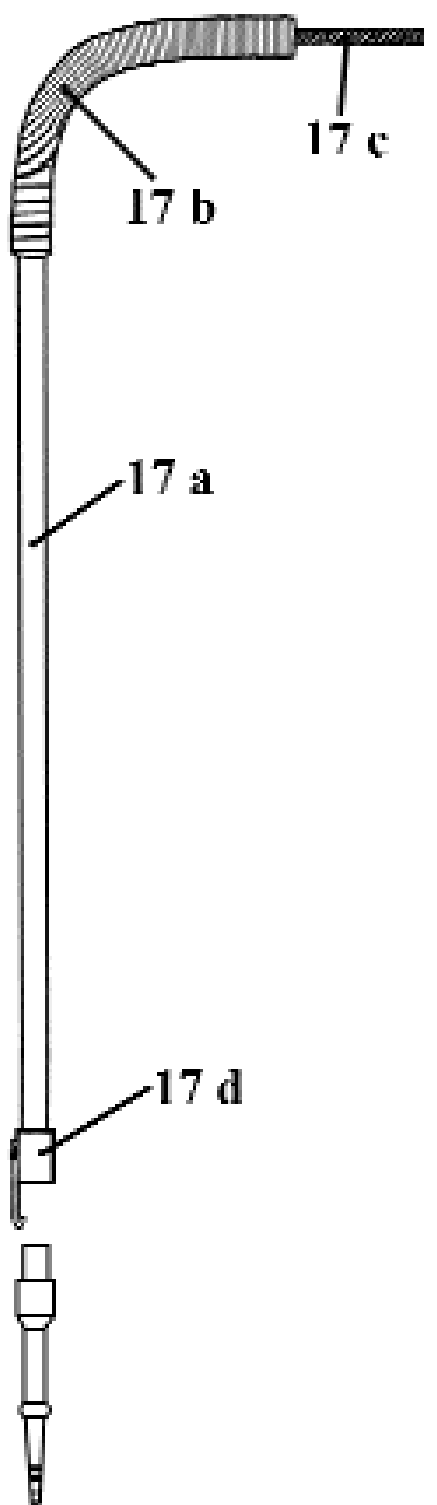

Figura 17: Sistema de cable: a) Latiguillo; b) Codera; c) Cable; d) Sistema de conexión.

d) que, alojado en el brazo horizontal, transmite el giro de la polea a la pieza de mano por medio de un cable (fig. 16 e) con el que es solidario.

\section{Polea secundaria}

Rueda metálica giratoria con garganta en su periferia (fig. 16 f), que pudiéndose deslizar en el canal del eje acanalado, recibe el movimiento giratorio procedente del volante, por medio de la correa de transmisión para comunicarlo al eje acanalado. La libertad de deslizamiento de la polea en el canal del eje acanalado es para compensar las distintas longitudes del cable como consecuencia de las flexiones que experimenta en las distintas posiciones de trabajo.

\section{Sistema de cable}

Originariamente (como se puede apreciar en la patente de Morrison), el movimiento de giro desarrollado en el brazo horizontal era transmitido por medio de un sistema de poleas a la pieza de mano.

Posteriormente, las poleas fueron sustituidas por un cable, que girando en el interior de un latiguillo o manguito semi-rígido, conducía dicho movimiento hasta la pieza de mano.

Al ser el sistema de cable el más frecuente, lo describimos a continuación. Un sistema de cable está constituido por:

- Latiguillo = manguito semirígido que unido en su extremo superior con el extremo libre del brazo horizontal, aloja en su interior al cable, protegiendo el giro de dicho cable (fig. 17 a)

- Codera = protector metálico flexible situado sobre la unión del brazo horizontal y el latiguillo, que protege el punto de unión de la parte flexible del cable con el eje (fig. 17 b).

- Cable = elemento flexible de acero, que alojado en el interior del latiguillo, hace llegar el movimiento giratorio del eje acanalado, con el cual es solidario, al sistema de conexión de la pieza de mano (fig. 17 c).

- Sistema de conexión = es el ele- mento encargado de la unión de la pieza de mano con el latiguillo y el cable que gira en su interior. La pieza de mano se acopla con el sistema de conexión y esta unión, a su vez, se fija por medio de un sistema de uña y muesca (fig. $17 \mathrm{~d}$ ).

\section{Mejoras}

Entre las mejoras incorporadas a la idea primitiva de Morrison cabrían destacar las siguientes:

- Uña retráctil en uno de los brazos delanteros del trípode o peana para mantener inclinado el torno.

- Apéndices de transporte solidarios al soporte (en lugar de a la varilla).

- Bomba para martillo de orificar.

- Cepillo limpia-fresas, situado en el eje de la polea secundaria.

- Soportes guías de la correa en la bisagra.

- Posibilidad de inclinación de la varilla y su fijación.

- Contrapeso en el extremo de la barra horizontal (modificación de Hastings).

- Variación de volante horizontal (Campbell).

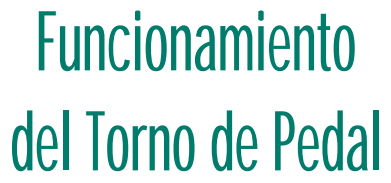

Todos estos elementos descritos, constituyen una máquina (Dental Engine) que en términos generales pesa entre 12 y $14 \mathrm{Kg}$ y tiene una altura de entre 1,20 y $1,40 \mathrm{~m}$ y que es capaz de desarrollar unas 800 vueltas por minuto con una pedalada por segundo, lo que fácilmente se puede demostrar por medio del siguiente cálculo:

Considerando que el desarrollo de 
la circunferencia del volante, es de Im aproximadamente y que la circunferencia de la polea secundaria es de $7 \mathrm{~cm}$, medidos ambos en la profundidad de sus respectivas gargantas, resulta que cada vuelta del volante genera aproximadamente 14 vueltas en la polea secundaria, y como quiera que el ritmo normal de pedaleo es de una pedalada por segundo, se puede estimar en 800 el número de vueltas que realiza la fresa por minuto.
Aunque existe un dispositivo adicional, que acoplado entre el extremo de la conexión y la pieza de mano, es capaz de multiplicar por dos el número de las revoluciones de la fresa.

\section{Agradecimientos}

A la Dra. Mạ Luisa Gozalvo, por su aportación de un torno de pedal portátil con su maleta.
A la Dra. Fabiola Kessler Nieto, por su traducción de alemán.
Al Dr. Miguel Aldecoa, ingeniero naval experto en mecánica, por su asesoramiento técnico.
A la Dra. Mạ. José López Silva, por su aportación de imágenes.
A todos los compañeros que han prestado sus tornos para este estudio.

\section{Bibliografía recomendada}

Para profundizar en la lectura de este tema, el/los autor/es considera/an interesantes los artículos que aparecen señalados del siguiente modo: *de interés **de especial interés.

1. Hoffmann - Axthelm W. et cols. Die Geschichte der Zahnheilkunde. Berlin: Quintessenz, 1973:292-3.

2. Dental Supplies Catalogue. Consolidated Dental Manufactures. Co. 1914:69-70.
3. Smith Lee S. Dental Catalogue. Lee S. Smith \& Son Co, 1915:231-2.

4. Ash C. Claudius Ash Catalogue. Claudius Ash et Fils, 1895:187.

5. Stehr F. Catálogo Ilustrado. F. Stehr - Trieste, 1888:52-4.
6. Windler H. Haupt Katalog. H. Windler, 1912: 250-1.

7. Durande Avellanal C. Diccionario Odontológico. Buenos Aires: Ediar S.A. Editores, 1955:698.

RCOE, 2003, Vol 8, №3, 305-314 